\title{
Skincare in Social Media: Analyzing Prominent Themes in Online Dermatologic Discussions
}

\author{
Aakash Reddy ${ }^{1}$ \\ 1. College of Natural Sciences, University of Texas at Austin, Austin, USA
}

Corresponding author: Aakash Reddy, reddyaakash01@utexas.edu

\begin{abstract}
Introduction: As the size and influence of online dermatologic communities have developed significantly in recent years, literature concerning the relationship between social media and dermatology grows more important. One community of interest is r/SkincareAddiction, a forum of over 1.2 million members on Reddit that serves as both a support group and discussion aggregator sourcing information from multiple social media platforms.
\end{abstract}

Methods: This study reports on the qualitative analysis of 300 highly engaged posts to identify prominent themes in online dermatologic discussions and determine the extent of multiplatform interaction between Reddit and other dermatology-relevant social networks.

Results: Results of an inductive analysis indicate discussions place an emphasis on communicating advice and improving general wellbeing, comprising $71 \%$ of measured posts. However, a large portion of this counsel lacks evidence and pseudoscientific recommendations are often accepted as factual. Additionally, $31 \%$ of entries were drawn from alternative social media sites.

Conclusion: Assessing the prevalent points of discussion in dermatology-relevant communities can inform clinical practice and reveal alternative methods to advance the delivery of care. As patients increasingly seek health advice and support online, developing digital literacy in common dermatologic trends offers a unique opportunity to improve communication, disseminate evidence-based counsel, and combat misinformation in both clinical and online settings.

Review began 04/24/2021 Review ended 04/30/2021 Published 05/07/2021

\section{(๑) Copyright 2021}

Reddy. This is an open access article distributed under the terms of the Creative Commons Attribution License CC-BY 4.0., which permits unrestricted use, distribution, and reproduction in any medium, provided the original author and source are credited.

Categories: Dermatology

Keywords: social media, reddit, skin health, online dermatology, skincare

\section{Introduction}

A growing body of literature is emphasizing the constantly evolving relationship between social media and dermatology [1-5]. As platforms such as Facebook and Instagram are used more frequently by patients seeking health advice and support on dermatologic conditions, literacy in online dermatologic trends becomes more important to improve communication, combat misinformation, and improve patient health outcomes [3,4]. In particular, communication between dermatologist and patient has been established as being crucial for effective diagnosis and treatment [6].

Online dermatologic forums on Reddit, the self-styled "front page of the internet", often discuss general skin care trends. One such message board is the subreddit titled r/SkincareAddiction where participants interact with large numbers of posts through commenting or voting. Boasting a community of 1.2 million anonymous members, the forum focuses on general skin health, cosmetic advice, and other dermatologic phenomenon [3]. Many of the most popular posts on $r$ /SkincareAddiction are cross-posted from other social media platforms such as Twitter, Instagram, and Tumblr. Consequently, the site functions as a discussion aggregator sourcing content across the internet, indicating Reddit incorporates a diverse cast of participants from the online social sphere. This study intends to classify and analyze popular points of discussion and dermatologic trends on Reddit.

\section{Materials And Methods}

The framework approach uses a standardized procedure in thematic analyses of qualitative data and enhances methodological transparency by employing explicitly defined stages of analysis [7]. The approach in practice involved investigating the 300 most upvoted posts in the subreddit $r$ /SkincareAddiction for patterns in text that were then inductively coded. Each code was categorized through an assignment to broader qualitative themes that were constantly refined through each stage of the process. Although some posts may be able to be classified in more than one code, posts were categorized into the one they most accurately represent as a whole. Usernames were not included to ensure the anonymity of posters was preserved. 
The data was analyzed through three stages [7]. The first, data management, involved the identification and assignment of initial codes from posts in the subreddit to a coding matrix. The second, descriptive accounts, involved the refinement of initial codes and themes and the formation of conceptual connections. The final stage, explanatory accounts, involved drawing general conclusions, relating associations between themes, and reflecting on the original data. In the first stage, the data was catalogued into a coding matrix comprised of five columns: post text, post description, In-vivo code, preliminary impressions, and initial code. Post descriptions and In-vivo codes (phrases taken from post text) were used to illustrate key concepts to ensure the voice of the original poster was being represented accurately [7]. Relevant initial codes were then grouped together into general themes in a coding index. Throughout the investigative process, both coding models were constantly refined to reflect the emergence of new patterns and associations. Increasingly nuanced depictions of the data developed as new insights were drawn between initial codes. In the final stages, broader concepts were developed into conclusions that reflected the data as a whole. Final themes were evaluated against initial data to ensure an accurate representation of the original text and prevent misinterpretation during the refinement process.

Additionally, sample size is considered sufficient when the point of saturation has been reached. Inductive thematic saturation for qualitative data is dependent on the number and frequency of new codes. Redundant codes indicate saturation has been established [8]. Consequently, 300 posts offer a sufficient dataset to draw preliminary codes from the most engaged points of discussion in the subreddit.

\section{Results}

Of the top 300 posts in the forum $\mathrm{r} /$ SkincareAddiction, four major themes emerged: i) advice on routines and products (33\%), ii) general discussions of skin health and wellbeing (39\%), iii) impact of skin ailments and their treatment options (14\%), and iv) social concerns in relation to self-image, media representations, and the skincare community (14\%). Results are listed in Table 1 . Each theme emerged by classifying a total of twelve refined codes that were initially in-vivo descriptions of the original text. For posts that disseminated advice, the expressed intent was to improve mental health and general wellbeing, together comprising $71 \%$ of measured posts. 


\section{Cureus}

\begin{tabular}{|c|c|c|}
\hline Theme & $\#$ & Code \\
\hline \multirow{3}{*}{ Advice } & 1 & General \\
\hline & 2 & Skincare Routine \\
\hline & 3 & Product Recommendations \\
\hline \multirow[t]{3}{*}{ Skin Health } & 4 & General Wellbeing \\
\hline & 5 & $\begin{array}{l}\text { Showcasing Satisfaction in Personal } \\
\text { Skin Health }\end{array}$ \\
\hline & 6 & Sharing Personal Struggles \\
\hline \multirow{3}{*}{$\begin{array}{l}\text { Effects on } \\
\text { Skin }\end{array}$} & 7 & Acne \\
\hline & 8 & Scarring \\
\hline & 9 & Sun \\
\hline \multirow[t]{2}{*}{ Social } & 10 & Self-image \\
\hline & 11 & Gender Bias \\
\hline & 12 & $\mathrm{~mm}$ \\
\hline
\end{tabular}

Code Description and Examples

General product safety, hygiene recommendations, techniques to break harmful skin habits.

Routine types and procedure, frequency of routine, description of personal regimen

Suggestions in favor of and against specific skincare products and companies.

Emphasize mental health, community support and understanding, sharing narratives of improvement

Selfies displaying skin, before and after pictures depicting transformations in skin.

Discussing frequent issues agitating community members, venting frustrations.

Treatment options, showcasing successful treatments, inquiries of product types.

General discussions of scarring, Acne scars, miscellaneous facial scarring.

Sunburns, skin reactions to variable weather, expressing the necessity of sunscreen.

Factors influencing self-esteem, reflections on media representations of skincare

Discussions of gendered marketing strategies, relationship between gender identity and skin care.

Material interactions between members of the community, care packages for healthcare workers.

TABLE 1: Inductive coding of the top 300 posts in r/SkincareAddiction

Additionally, $31 \%$ of posts were cross-posted across multiple social media platforms. Of these, 44 are from Twitter, 43 are from Instagram, four are from TikTok, and three are from Tumblr. Posts from Twitter and Instagram were $47 \%$ and $46 \%$ of cross-posts respectively. On the other hand, posts from TikTok (4\%) and Tumblr (3\%) were a notably smaller proportion. The extent of the subreddit's multiplatform interactions is recorded in Table 2 . Ten posts directly referenced dermatologic practices such as by recommending a consultation, passing on advice from a provider, or disseminating social media posts by dermatologists.

\begin{tabular}{|c|c|c|c|}
\hline Platform & Cross-posts & $\%$ of total posts & $\%$ of cross-posts \\
\hline Twitter & 44 & $15 \%$ & $47 \%$ \\
\hline Instagram & 43 & $14 \%$ & $46 \%$ \\
\hline TikTok & 4 & $1 \%$ & $4 \%$ \\
\hline Tumblr & 3 & $1 \%$ & $3 \%$ \\
\hline Total & 94 & $31 \%$ & \\
\hline
\end{tabular}

TABLE 2: Posts from alternative platforms

\section{Discussion}

Online forums such as r/SkincareAddiction form tight-knit communities to express grievances, share 
personal stories, and develop unique points of discussion. As users credit the subreddit with significantly improving their knowledge of skin health, many posts take the form of counsel to others as a way to pass on shared knowledge. As members of online forums frequently consult social media for dermatologic information, advice posts may become more influential in the decision-making patterns of dermatologic patients [3]. While general advice was within an atmosphere of mutual learning, the advice in question varied widely from hygienic techniques (wash pillowcases frequently) to breaking habits (stop touching your face). However, advice relevant to skincare routines directly associated a disciplined routine with stability and organization. Regardless of the nature of the practice, simply having a skincare regimen done on a consistent basis was a regulatory force for many posters. For advice on skincare products and companies, some incorporated ethical concerns such as predatory corporations and labor abuse.

The largest number of posts were coded under general wellbeing and related skin health to security, happiness, and fulfillment. Members of the community argue their focus is beyond just appearance, indicating that skincare is a catalyst for general health and wellness. In particular, posters associate skin health with their mood as one poster expressed distress "when I wake up with a pimple”. Conversely, posts of individuals showcasing satisfaction with their skin were accompanied by positive remarks on their disposition ("self-esteem is back"). When posters feel frustrated, however, they are more likely to share their struggles, venting to the community. The subreddit serves as a support group through communicating problems and encouraging other members ("Your skin is NOT disgusting").

When concerned with the specifics of skin health, posters emphasize the effects of particular ailments on their skin. Posts coded under 'sun' are generally discussions noting the utility of sunscreen and the dangers of uninterrupted sunlight. For example, one poster described their experience consistently applying sunscreen for years, noting how they felt significant improvements in physical appearance compared to when they had not used sunscreen at all. Posts coded under acne, however, tend to be of transformations (i.e. before and after pictures) and successful treatments. For some posters, acne was associated with depression ("defeated fungal acne... and depression"), reflecting the extensive literature associating acne with depression in dermatology patients [9-13]. Posts on scarring were also frequently related to acne, such as a request for advice on removing acne scars or a successful acne scar treatment. Many members of the community lamented the visual effects of both acne and acne scarring on their physical appearance. Posters discussing scarring also tended to depict transformations using before and after pictures, recognizing improvements in self-esteem $[14,15]$. Self-confidence can also be negatively affected through what posts identify as unrealistic beauty standards in media ("it's just a whole lot of...photoshop") and use the forum as a mechanism to combat the pervasiveness of media representations. The community condemns marketing campaigns that display heavily edited photos of skin. Gendered marketing strategies often play into these depictions as numerous posts reflect on how their gender informs interactions with skincare companies and products [16]. In response, the subreddit rallied around building a skin positive community with the goal of showcasing imperfections and encouraging the reconstruction of a healthy self-image. Reflecting a prior study on online dermatologic communities, shared struggles appear to link the subreddit's members together and strengthen communal bonds through mutual aid and support [17]. Of cross-posts from alternative social media platforms, the majority were from Twitter and Instagram, sites that are frequently used by dermatology-relevant groups [18]. The large reach of Reddit incentivizes the accumulation of discussions from a wide range of sources. Consequently, just under $1 / 3$ of the top 300 posts are from supplementary social networks.

Due to the nature of qualitative analysis, methodological limitations include subjectivity and lack of transparency [19]. In addition, posts from mass communication platforms are not primed for research. As a result, it is important to approach the coding matrix with caution to retain a strong basis in the text and avoid overstating conclusions. The framework approach was used to counter these limitations by highlighting the procedure of qualitative coding, identifying the stages of analysis, and using in-vivo text as initial codes.

\section{Conclusions}

The vast size and influence of r/SkincareAddiction offer a compelling rationale for improving digital literacy in online dermatologic trends. The forum's accumulation of posts from multiple social media platforms animates the subreddit as a vehicle for guidance, support, and dialogue. Although these resources can educate patients, misinformation regarding treatments and product sourcing can be equally rampant. Through accounting for popular points of discussion, general inaccuracies, and common themes on dermatologic social media, the information explored in this project can aid in developing a shared physicianpatient vocabulary in online and clinical settings. In consideration of previous literature, future research can emphasize distinctions between dermatologic social media platforms and assess the comparative relevance between online discussions and successful clinical outcomes.

\section{Additional Information}

\section{Disclosures}

Human subjects: All authors have confirmed that this study did not involve human participants or tissue. Animal subjects: All authors have confirmed that this study did not involve animal subjects or tissue. 
Conflicts of interest: In compliance with the ICMJE uniform disclosure form, all authors declare the following: Payment/services info: All authors have declared that no financial support was received from any organization for the submitted work. Financial relationships: All authors have declared that they have no financial relationships at present or within the previous three years with any organizations that might have an interest in the submitted work. Other relationships: All authors have declared that there are no other relationships or activities that could appear to have influenced the submitted work.

\section{References}

1. DeBord LC, Patel V, Braun TL, Dao H Jr: Social media in dermatology: clinical relevance, academic value, and trends across platforms. J Dermatolog Treat. 2019, 30:511-8. 10.1080/09546634.2018.1530444

2. Buntinx-Krieg T, Caravaglio J, Domozych R, Dellavalle RP: Dermatology on Reddit: elucidating trends in dermatologic communications on the world wide web. Dermatol Online J. 2017, 23:2.

3. Schoenberg E, Shalabi D, Wang JV, Saedi N, Keller M: Public social media consultations for dermatologic conditions: an online survey. Dermatol Online J. 2020, 26:6.

4. Parks R, Newsom EC, Park JH, Lawrence N: Skincare addiction on Reddit: dermatology enthusiasts talk skin . Dermatol Surg. 2020, 46:1372-74. 10.1097/DSS.0000000000002060

5. Jowett S, Ryan T: Dermatology patients and their doctors. Clin Exp Dermatol. 1985, 10:246-54. 10.1111/j.1365-2230.1985.tb00565.x

6. Smith J, Firth J: Qualitative data analysis: the framework approach . Nurse Res. 2011, 18:52-6. 10.7748/nr2011.01.18.2.52.c8284

7. Petukhova TA, Wilson BN, Gadjiko M, Lee EH, Wang J, Rossi AM, Nehal KS: Utilization of Facebook for support and education by patients with skin cancer. Dermatol Online J. 2020, 26:4.

8. Saunders B, Sim J, Kingstone T, et al.: Saturation in qualitative research: exploring its conceptualization and operationalization. Qual Quant. 2018, 52:1893-907. 10.1007/s11135-017-0574-8

9. Samuels DV, Rosenthal R, Lin R, Chaudhari S, Natsuaki MN: Acne vulgaris and risk of depression and anxiety: a meta-analytic review. J Am Acad Dermatol. 2020, 83:532-41. 10.1016/j.jaad.2020.02.040

10. Öztekin C, Öztekin A: The association of depression, loneliness and internet addiction levels in patients with acne vulgaris. BioPsychoSocial Med. 2020, 14:17. 10.1186/s13030-020-00190-y

11. Singh N, Singh A, Pandey K, Nimisha: Current insights for the management of acne in the modern era . Recent Pat Antiinfect Drug Discov. 2020, 15:3-29. 10.2174/1574891X15999200729192138

12. Peng T, Li ZM, Liu J, Zhang Y: Evaluation of reliability and validity of the Patient Health Questionnaire-9 in patients with acne. Dermatol Ther. 2020, 33:e13584. 10.1111/dth.13584

13. Altunay IK, Özkur E, Dalgard FJ, et al.: Psychosocial aspects of adult acne: data from 13 European countries . Acta Derm Venereol. 2020, 100:10.2340/00015555-3409

14. Aslan Kayiran M, Karadag AS, Jafferany M: Psychodermatology of acne: dermatologist's guide to inner side of acne and management approach. Dermatol Ther. 2020, 33:e14150. 10.1111/dth.14150

15. Motoki THC, Isoldi FC, Brito MJA, Filho AG, Ferreira LM: Keloid negatively affects body image . Burns. 2019, 45:610-4. 10.1016/j.burns.2018.10.009

16. Petit A: Skin lightening and its motives: a historical overview . Ann Dermatol Venereol. 2019, 146:399-40. 10.1016/i.annder.2019.04.017

17. Ross NA, Todd Q, Saedi N: Patient seeking behaviors and online personas: social media's role in cosmetic dermatology. Dermatol Surg. 2015, 41:269-76. 10.1097/DSS.0000000000000267

18. Karimkhani C, Connett J, Boyers L, Quest T, Dellavalle RP: Dermatology on Instagram. Dermatol Online J. 2014, 20:

19. Attride-Stirling J: Thematic networks: an analytic tool for qualitative research. Qual Res. 2001, 1:385-405. $10.1177 / 146879410100100307$ 